\title{
Clasificación GOLD 2011 de la enfermedad pulmonar obstructiva crónica: un paso adelante, pero aún atrás
}

A finales de diciembre de 2011, apareció en la web la nueva versión de la Guía GOLD (del inglés Global Initiative for Chronic Obstructive Lung Disease) para el diagnóstico, manejo y prevención de la enfermedad pulmonar obstructiva crónica y, transcurridos diez años desde la publicación de la primera versión, esta supone un cambio fundamental en lo que respecta a los objetivos del tratamiento de la enfermedad y a la evaluación y clasificación de la gravedad del paciente (1).

La guia GOLD 2011 resume en dos los varios objetivos terapéuticos propuestos en las versiones anteriores: 1 . Minimizar o evitar el impacto de los síntomas sobre la salud del paciente y 2 . Disminuir el riesgo futuro de eventos adversos como exacerbaciones o mortalidad (1).

Este planteamiento tiene un efecto directo en la propuesta de valoración clínica del paciente con enfermedad pulmonar obstructiva crónica pues supone una evaluación multidimensional en concordancia con dichos objetivos y ya no exclusiva en el VEF1; de tal forma, ahora se propone valorar el impacto de los síntomas sobre el estado de salud del paciente, empleando la escala modificada de disnea del Medical Research Council (mMRC) o el cuestionario CAT (COPD Assessment Test), y el riesgo de exacerbaciones, utilizando la clasificación espirométrica ya conocida de limitación al flujo aéreo con el VEF, postbroncodilatador y la frecuencia de exacerbaciones en el último año, empleando aquella de las dos que se asocie a un riesgo superior (2-4).

La evaluación combinada sitúa al paciente en cuatro categorias según la intensidad actual de sus síntomas y su riesgo futuro de exacerbaciones (1):

A: menos síntomas ( $m M R C 0-1$ o CAT $\leq 10$ ) y bajo riesgo $\left(V_{E F} \geq 50 \%\right.$ ).

$B$ : más síntomas ( $m M R C \geq 2$ o CAT $\geq 10$ ) y bajo riesgo $\left(V E F_{1} \geq 50 \%\right.$ ).

C: menos sintomas ( $m M R C 0-1$ o CAT $\leq 10)$ y alto riesgo $\left(V_{1} F_{1}<50 \%\right)$.

$D$ : más síntomas ( $m M R C \geq 20 C A T \geq 10)$ y alto riesgo $\left(V E F_{1}<50 \%\right)$.

Esta nueva forma de evaluación del paciente con enfermedad pulmonar obstructiva crónica recoge la idea propuesta por López-Campos (5) y se construyó por consenso extrapolando información ya conocida sobre la capacidad pronóstica de la escala mMRC, la evidencia disponible sobre el cuestionario CAT y los datos de tres estudios clínicos en enfermedad pulmonar obstructiva crónica (TORCH, UPLIFT y ECLIPSE) (1-4).

La nueva clasificación tiene más sentido clínico, ya que las decisiones de tratamiento no deberian estar basadas sólo en la limitación al flujo aéreo, sino en otras evaluaciones relevantes y no precisa mediciones sofisticadas, por lo que puede ser aplicada en cualquier situación clínica y lugar y sitúa el tratamiento de la enfermedad pulmonar obstructiva crónica en el marco de una práctica más cercana a las necesidades individuales de cada paciente (2-4).

Desde la publicación de la guía GOLD 2011, tres estudios han evaluado las diferencias con la clasificación previa y su capacidad pronóstica (6-8).

Soriano y colaboradores llevaron a cabo un análisis en 3.633 pacientes de los más de 11.000 del estudio COCOMICS (del inglés COllaborative COhorts to assess Multicomponent Indices of COPD in Spain) que incluye los datos de pacientes individuales de once cohortes españolas de enfermedad pulmonar obstructiva crónica, con el fin de determinar la distribución de las nuevas categorías y su habilidad pronóstica para predecir mortalidad en comparación con la clasificación previa (6).

Según esta última, la distribución de los pacientes fue enfermedad pulmonar obstructiva crónica leve 10,2\%, moderada $44,8 \%$, severa $34,9 \%$, muy severa $10,2 \%$, mientras que con la nueva categorización $33,6 \%$ fueron grupo $A, 16,3 \%$ grupo $B, 17,7 \%$ grupo $C$ y $32,3 \%$ grupo $D$, con gran heterogeneidad de ambas clasificaciones dentro de las cohortes. No se observaron diferencias entre las dos clasificaciones en la capacidad para predecir mortalidad a 1, 3 ó 10 años, y tampoco entre los grupos B y C (6). 